\title{
Existence and uniqueness of solutions to discrete, third-order three-point boundary value problems
}

\author{
Saleh S. Almuthaybiri ${ }^{1,2}$ \\ JAGAN MOHAN JONNALAGADDA ${ }^{3}$ \\ Christopher C. Tisdell ${ }^{1}$ \\ 1 School of Mathematics and Statistics, \\ The University of New South Wales, \\ UNSW, Sydney, NSW, 2052, Australia. \\ cct@unsw.edu.au \\ 2 Department of Mathematics, College of \\ Science and Arts in Uglat Asugour, \\ Qassim University, Buraydah, Kingdom of \\ Saudi Arabia. \\ s.almuthaybiri@qu.edu.sa \\ 3 Department of Mathematics, Birla \\ Institute of Technology and Science Pilani, \\ Hyderabad, 500078, Telangana, India. \\ j.jaganmohan@hotmail.com
}

\begin{abstract}
The purpose of this article is to move towards a more complete understanding of the qualitative properties of solutions to discrete boundary value problems. In particular, we introduce and develop sufficient conditions under which the existence of a unique solution for a third-order difference equation subject to three-point boundary conditions is guaranteed. Our contributions are realized in the following ways. First, we construct the corresponding Green's function for the problem and formulate some new bounds on its summation. Second, we apply these properties to the boundary value problem by drawing on $\mathrm{Ba}$ nach's fixed point theorem in conjunction with interesting metrics and appropriate inequalities. We discuss several examples to illustrate the nature of our advancements.

RESUMEN

El propósito de este artículo es avanzar hacia un entendimiento más completo de las propiedades cualitativas de las soluciones a problemas discretos de valor en la frontera. En particular, introducimos y desarrollamos condiciones suficientes bajo las cuales se garantiza la existencia de una única solución para una ecuación en diferencias de tercer orden sujeta a condiciones de borde en tres puntos. Nuestras contribuciones son de dos tipos. En primer lugar, construimos las funciones de Green correspondientes para el problema y formulamos nuevas cotas para su suma. En segundo lugar, aplicamos estas propiedades al problema de valor en la frontera usando el teorema del punto fijo de Banach junto con métricas interesantes y desigualdades apropiadas. Discutimos varios ejemplos para ilustrar la naturaleza de nuestros avances.
\end{abstract}

Keywords and Phrases: Forward difference, boundary value problem, Green's function, contraction, fixed point, existence, uniqueness.

2020 AMS Mathematics Subject Classification: 39A12.

\section{(cc) BY-NC}




\section{Introduction}

Discrete boundary value problems are of significant interest to scientific and technical communities. For instance, their perceived utility is partly due to their ability to act as a mathematical framework to model purely discrete processes and phenomena that arise in various fields of science and engineering. In addition, developing a theory of discrete boundary value problems has the potential to inform our understanding of continuous boundary value problems. For example, discrete boundary value problems can arise as approximations to "continuous" boundary value problems that involve differential equations, where the numerical aspects of solutions are of importance. Furthermore, it is also possible to construct a theory of differential equations by only using difference equations [7].

Although discrete problems have enjoyed continued interest, the mathematics community is yet to reach a complete understanding of the qualitative and quantitative properties of their solutions. This includes, for example, discrete boundary value problems of the third order, which have not been advanced to the same degree as their "continuous cousins" or to the same extent as discrete problems of the second order. Moreover, we are yet to achieve a total comprehension of the mathematical similarities and distinctions between such continuous and discrete problems.

Motivated by the above discussion, the purpose of the current paper is to make progress towards a more complete theory concerning the existence and uniqueness of solutions to discrete boundary value problems of the third order. "Knowing an equation has a unique solution is important from both a modelling and theoretical point of view" [19, p. 794] as it informs our mathematical understanding from applied and pure perspectives. For example, by developing a deeper understanding of the existence and uniqueness of solutions to discrete boundary value problems we are simultaneously expanding capacity and knowledge of the associated models and the mathematical frameworks that attempt to describe them.

For any $a, b \in \mathbb{R}$ such that $(b-a) \in \mathbb{N}$, we will denote $\mathbb{N}_{a}=\{a, a+1, a+2, \ldots\}$ and $\mathbb{N}_{a}^{b}=$ $\{a, a+1, a+2, \ldots, b\}$. Let $\Delta$ denote the usual forward difference operator defined by

$$
(\Delta u)(t)=u(t+1)-u(t), \quad t \in \mathbb{N}_{0}^{T+2},
$$

Herein we will consider the following third-order, three-point discrete boundary value problem

$$
\left\{\begin{array}{l}
\left(\Delta^{3} u\right)(t-2)+f(t, u(t))=0, \quad t \in \mathbb{N}_{2}^{T+2}, \\
u(0)=(\Delta u)(0)=0, \quad u(T+3)=k u(\eta)
\end{array}\right.
$$

where $f$ is a continuous function from $\mathbb{N}_{0}^{T+3} \times \mathbb{R}$ to $\mathbb{R}$ which we denote via $f \in C\left[\mathbb{N}_{0}^{T+3} \times \mathbb{R}, \mathbb{R}\right]$. In addition, $T \in \mathbb{N}_{1}, k \in \mathbb{R}$ and $\eta \in \mathbb{N}_{1}^{T+2}$.

Let us briefly outline recent and relevant literature to situate and contextualize our work. Agarwal and Henderson [2] initiated the study of positive solutions to the third-order three-point discrete 
boundary value problem

$$
\left\{\begin{array}{l}
\left(\Delta^{3} u\right)(t-2)+a(t) g(u(t))=0, \quad t \in \mathbb{N}_{2}^{T+2} \\
u(0)=u(1)=u(T+3)=0
\end{array}\right.
$$

where $a: \mathbb{N}_{0}^{T+2} \rightarrow \mathbb{R}^{+}$and $g \in C\left[\mathbb{R}^{+}, \mathbb{R}^{+}\right]$. Following this work, Anderson [5] and Anderson and Avery [6] examined the existence of multiple solutions to third-order, three-point discrete focal boundary value problems. Positive solutions to discrete, third-order problems have been shown to exist using fixed point theory in cones [13]. In addition, several authors have discussed various qualitative properties of different classes of third-order three-point discrete boundary value problems and a detailed discussion can be found in $[11,12,23,24,25,13]$ and the references therein.

Motivated by the recent work [4, 15], where the differential equation version of (1.1) was analyzed, in the present article we investigate the discrete boundary value problem (1.1). When compared with the ideas in $[4,15]$ our methods and results herein are different; and they reveal some thoughtprovoking distinctions and connections between the sets of works. For example, the present work develops alternative bounds on the Green's functions to those in $[4,15]$ and we employ purely discrete ways of working. In particular, we observe that some of our bounds for the discrete case are sharp, while others are rougher. The bounds are different from those developed for the continuous case [4]. This highlights some of the interesting distinctions between the discrete and the continuous in terms of results and methods within the domain of third order problems.

Our article is organized as follows: In Section 2, we construct the Green's function corresponding to the boundary value problem (1.1) and establish new bounds on its summation. In Section 3 we apply the properties of the Green's function to the boundary value problem (1.1) in conjunction with Banach's contraction mapping theorem to establish sufficient conditions for the existence of a unique solution. We provide a discussion of examples in Section 4 to illustrate how our ideas can be put into practice and the relationships between them. Finally, we conclude with some ideas for further work in Section 5.

For more on discrete problems, see the monographs $[1,8,9,10,14]$.

\section{Green's function and its properties}

In order to develop the Green's function for the three-point case, we first analyze the two-point discrete boundary value problem

$$
\left\{\begin{array}{l}
\left(\Delta^{3} v\right)(t-2)+h(t)=0, \quad t \in \mathbb{N}_{2}^{T+2} \\
v(0)=(\Delta v)(0)=v(T+3)=0
\end{array}\right.
$$


where $h \in C\left[\mathbb{N}_{2}^{T+2}, \mathbb{R}\right]$. The boundary value problem (2.1) can be equivalently rewritten as

$$
\left\{\begin{array}{l}
\left(\Delta^{3} v\right)(t-2)+h(t)=0, \quad t \in \mathbb{N}_{2}^{T+2} \\
v(0)=v(1)=v(T+3)=0
\end{array}\right.
$$

Yang and Weng [25] derived a Green's function for the boundary value problem (2.2) and also investigated its sign. The following two results are found therein and will be helpful in our present analysis.

Lemma 2.1 ([25]). The unique solution of the boundary value problem (2.2) (or (2.1)) is given by

$$
v(t)=\sum_{s=2}^{T+2} H(t, s) h(s), \quad t \in \mathbb{N}_{0}^{T+3}
$$

where

$$
H(t, s)= \begin{cases}\frac{t(t-1)(T+3-s)(T+4-s)}{2(T+3)(T+2)}-\frac{(t-s)(t-s+1)}{2}, & s \in \mathbb{N}_{0}^{t-1}, \\ \frac{t(t-1)(T+3-s)(T+4-s)}{2(T+3)(T+2)}, & s \in \mathbb{N}_{t}^{T+3} .\end{cases}
$$

Lemma 2.2 ([25]). The Green's function $H(t, s)$ in (2.4) satisfies $H(t, s) \geq 0$ for all $(t, s) \in$ $\mathbb{N}_{0}^{T+3} \times \mathbb{N}_{2}^{T+2}$.

Now let us construct the Green's function for the boundary value problem

$$
\left\{\begin{array}{l}
\left(\Delta^{3} u\right)(t-2)+h(t)=0, \quad t \in \mathbb{N}_{2}^{T+2}, \\
u(0)=(\Delta u)(0)=0, \quad u(T+3)=k u(\eta)
\end{array}\right.
$$

to form the following new result.

Lemma 2.3. Let $h \in C\left[\mathbb{N}_{2}^{T+2}, \mathbb{R}\right]$ and assume

$$
(T+2)(T+3) \neq k \eta(\eta-1) .
$$

The unique solution to the boundary value problem (2.5) is given by

$$
u(t)=\sum_{s=2}^{T+2} G(t, s) h(s), \quad t \in \mathbb{N}_{0}^{T+3}
$$

where

$$
G(t, s)=H(t, s)+\frac{k t(t-1)}{(T+2)(T+3)-k \eta(\eta-1)} H(\eta, s) .
$$

Proof. Assume the solution of the boundary value problem (2.5) can be expressed as

$$
u(t)=v(t)+\left[C_{0}+C_{1} t+C_{2} t(t-1)\right] v(\eta),
$$


where $C_{0}, C_{1}$ and $C_{2}$ are constants to be determined and $v$ be the unique solution of the boundary value problem (2.1). When $v(\eta)=0$, the $u(t)$ defined by (2.8) is the same as $v(t)$ and it is actually the solution of $(2.5)$. In what follows, we assume that $v(\eta) \neq 0$.

It follows from $(2.8)$ that

$$
(\Delta u)(t)=(\Delta v)(t)+\left[C_{1}+2 C_{2} t\right] v(\eta) .
$$

From (2.1), (2.5), (2.8) and (2.9), we have

$$
\begin{aligned}
& u(0)=0 \Rightarrow v(0)+C_{0} v(\eta)=0 \Rightarrow C_{0}=0, \\
& (\Delta u)(0)=0 \Rightarrow(\Delta v)(0)+C_{1} v(\eta)=0 \Rightarrow C_{1}=0,
\end{aligned}
$$

and

$$
\begin{aligned}
& u(T+3)=k u(\eta) \\
\Rightarrow & v(T+3)+C_{2}(T+2)(T+3) v(\eta)=k\left[v(\eta)+C_{2} \eta(\eta-1) v(\eta)\right] \\
\Rightarrow & C_{2}=\frac{k}{(T+2)(T+3)-k \eta(\eta-1)} .
\end{aligned}
$$

Using (2.3) and (2.10) - (2.12) in (2.8) and rearranging the terms, we obtain (2.6) and (2.7). The proof is complete.

Let us now establish new bounds on the summation of the Green's functions $H(t, s)$ and $G(t, s)$ via the following result, which is of interest in its own right, for example, the bounds may prove useful in areas beyond the scope of this paper, such as in the application of topological ways of working with fixed point theory. We will draw on it to establish the main existence and uniqueness results of Section 3. The bounds will be formulated in terms of $T, k, \eta$. To assist with notation, we define the following constant $\Lambda$ (that depends on the form of $T$ ) that we will use below. For $n \in \mathbb{N}_{1}$ we define

$$
\Lambda= \begin{cases}\frac{n(n+2)(2 n+1)}{3}, & \text { if } T=3 n, \\ \frac{n(n+1)(2 n+1)}{3}, & \text { if } T=3 n-1, \\ \frac{n(n+1)(2 n-1)}{3}, & \text { if } T=3 n-2 .\end{cases}
$$

Lemma 2.4. The Green's function $G(t, s)$ in (2.7) satisfies

$$
\sum_{s=2}^{T+2}|G(t, s)| \leq \Gamma,
$$

where $\Gamma$ depends on $T, k, \eta$ and is explicitly given by

$$
\Gamma=\Lambda+\left|\frac{k}{(T+2)(T+3)-k \eta(\eta-1)}\right|(T+2)(T+3)\left[\frac{\eta(\eta-1)(T+7)}{6}+\eta\right]
$$

and $\Lambda$ is defined in (2.13). 
Proof. Consider

$$
\begin{aligned}
\sum_{s=2}^{T+2} H(t, s)= & \sum_{s=2}^{t-1}\left[\frac{t(t-1)(T+3-s)(T+4-s)}{2(T+3)(T+2)}-\frac{(t-s)(t-s+1)}{2}\right] \\
& +\sum_{s=t}^{T+2}\left[\frac{t(t-1)(T+3-s)(T+4-s)}{2(T+3)(T+2)}\right] \\
= & \sum_{s=2}^{T+2}\left[\frac{t(t-1)(T+3-s)(T+4-s)}{2(T+3)(T+2)}\right]-\sum_{s=2}^{t-1}\left[\frac{(t-s)(t-s+1)}{2}\right] \\
= & \frac{t(t-1)}{2(T+3)(T+2)} \sum_{s=1}^{T} s(s+1)-\frac{1}{2} \sum_{s=1}^{t-2} s(s+1) \\
= & \frac{t(t-1)}{2(T+3)(T+2)}\left[\sum_{s=1}^{T} s^{2}+\sum_{s=1}^{T} s\right]-\frac{1}{2}\left[\sum_{s=1}^{t} s^{2}+\sum_{s=1} s\right] \\
= & \frac{t(t-1)}{2(T+3)(T+2)}\left[\frac{(T+1)(T+2)(2 T+3)}{6}+\frac{(T+1)(T+2)}{2}\right] \\
& -\frac{1}{2}\left[\frac{(t-2)(t-1)(2 t-3)}{6}+\frac{(t-2)(t-1)}{2}\right] \\
= & \frac{t(t-1)(T+1)-t(t-1)(t-2)}{6}=\frac{t(t-1)(T+3-t)}{6} .
\end{aligned}
$$

Clearly,

$$
\sum_{s=2}^{T+2} H(0, s)=\sum_{s=2}^{T+2} H(1, s)=0
$$

Now we wish to maximize $\sum_{s=2}^{T+2} H(t, s)$ for $t \in \mathbb{N}_{2}^{T+3}$. Denote by

$$
g(t)=\frac{t(t-1)(T+3-t)}{6}, \quad t \in \mathbb{N}_{2}^{T+3}
$$

The first forward difference of $g$ with respect to $t$ is given by

$$
(\Delta g)(t)=\frac{t(2 T-3 t+5)}{6} \text {. }
$$

In this expression, the term $t / 6$ is positive for all $t \in \mathbb{N}_{2}^{T+3}$. The equation $2 T-3 t+5=0$ has the solution $t=\frac{2 T+5}{3}$, so we consider $t=\left\lfloor\frac{2 T+5}{3}\right\rfloor \in \mathbb{N}_{2}^{T+3}$. If $t \leq\left\lfloor\frac{2 T+5}{3}\right\rfloor$, the difference $2 T-3 t+5$ is positive, and thus $g$ is increasing. If $t>\left\lfloor\frac{2 T+5}{3}\right\rfloor$, the quantity $2 T-3 t+5$ is negative, and thus $g$ is decreasing. Hence, the maximum value of $g$ occurs at $t=\left\lfloor\frac{2 T+5}{3}\right\rfloor$. We observe that, for $n \in \mathbb{N}_{1}$,

$$
\left\lfloor\frac{2 T+5}{3}\right\rfloor= \begin{cases}2 n+1, & \text { if } T=3 n \\ 2 n+1, & \text { if } T=3 n-1 \\ 2 n, & \text { if } T=3 n-2 .\end{cases}
$$

Therefore,

$$
\max _{t \in \mathbb{N}_{0}^{T+3}} \sum_{s=2}^{T+2} H(t, s)=\max _{t \in \mathbb{N}_{2}^{T+3}} g(t)=g\left(\left\lfloor\frac{2 T+5}{3}\right\rfloor\right)=\Lambda .
$$


Consider

$$
\begin{aligned}
\sum_{s=2}^{T+2} H(\eta, s)= & \sum_{s=2}^{t-1}\left[\frac{\eta(\eta-1)(T+3-s)(T+4-s)}{2(T+3)(T+2)}-\frac{(\eta-s)(\eta-s+1)}{2}\right] \\
& +\sum_{s=t}^{T+2}\left[\frac{\eta(\eta-1)(T+3-s)(T+4-s)}{2(T+3)(T+2)}\right] \\
= & \sum_{s=2}^{T+2}\left[\frac{\eta(\eta-1)(T+3-s)(T+4-s)}{2(T+3)(T+2)}\right]-\sum_{s=2}^{t-1}\left[\frac{(\eta-s)(\eta-s+1)}{2}\right] \\
= & \frac{\eta(\eta-1)}{2(T+3)(T+2)} \sum_{s=1}^{T+1} s(s+1)-\frac{1}{2} \sum_{s=1}^{t-2}(\eta-s)(\eta-s-1) \\
= & \frac{\eta(\eta-1)}{2(T+3)(T+2)}\left[\sum_{s=1}^{T+1} s^{2}+\sum_{s=1}^{T+1} s\right]-\frac{1}{2}\left[\eta(\eta-1) \sum_{s=1}^{t-2} 1-(2 \eta-1) \sum_{s=1}^{t-2} s+\sum_{s=1}^{t-2} s^{2}\right] \\
= & \frac{\eta(\eta-1)}{2(T+3)(T+2)}\left[\frac{(T+1)(T+2)(2 T+3)}{6}+\frac{(T+1)(T+2)}{2}\right] \\
& -\frac{1}{2}\left[\eta(\eta-1)(t-2)-(2 \eta-1) \frac{(t-2)(t-1)}{2}+\frac{(t-2)(t-1)(2 t-3)}{6}\right] \\
= & \frac{\eta(\eta-1)(T-3 t+7)+(3 \eta-t)(t-1)(t-2)}{6} .
\end{aligned}
$$

Now we wish to maximize $\sum_{s=2}^{T+2} H(\eta, s)$ for $t \in \mathbb{N}_{0}^{T+3}$. Denote by

$$
h(t)=\frac{\eta(\eta-1)(T-3 t+7)+(3 \eta-t)(t-1)(t-2)}{6}, \quad t \in \mathbb{N}_{0}^{T+3} .
$$

The first forward difference of $h$ with respect to $t$ is given by

$$
(\Delta h)(t)=-\frac{t^{2}-(2 \eta+1) t+\eta(\eta+1)}{2} .
$$

We observe that

$$
(\Delta h)(t) \begin{cases}<0, & \text { for } t \in \mathbb{N}_{0}^{\eta-1} \\ =0, & \text { for } t=\eta \\ =0, & \text { for } t=\eta+1 \\ <0, & \text { for } t \in \mathbb{N}_{\eta+2}^{T+3}\end{cases}
$$

implying that

$$
\max _{t \in \mathbb{N}_{0}^{T+3}} h(t)=h(0)=\frac{\eta(\eta-1)(T+7)}{6}+\eta
$$

That is,

$$
\max _{t \in \mathbb{N}_{0}^{T+3}} \sum_{s=2}^{T+2} H(\eta, s)=\frac{\eta(\eta-1)(T+7)}{6}+\eta
$$


Now, consider

$$
\begin{aligned}
\sum_{s=2}^{T+2}|G(t, s)| & =\sum_{s=2}^{T+2}\left|H(t, s)+\frac{k t(t-1)}{(T+2)(T+3)-k \eta(\eta-1)} H(\eta, s)\right| \\
& \leq \sum_{s=2}^{T+2}|H(t, s)|+\left|\frac{k}{(T+2)(T+3)-k \eta(\eta-1)}\right| t(t-1) \sum_{s=2}^{T+2}|H(\eta, s)| \\
& =\sum_{s=2}^{T+2} H(t, s)+\left|\frac{k}{(T+2)(T+3)-k \eta(\eta-1)}\right| t(t-1) \sum_{s=2}^{T+2} H(\eta, s) \\
& \leq \Lambda+\left|\frac{k}{(T+2)(T+3)-k \eta(\eta-1)}\right|(T+2)(T+3)\left[\frac{\eta(\eta-1)(T+7)}{6}+\eta\right] \\
& =\Gamma .
\end{aligned}
$$

The proof is complete.

Remark 2.5. From the proof of Lemma 2.4 we see that (2.15) implies that the bound $\Lambda$ on

$$
\sum_{s=2}^{T+2} H(t, s), \quad t \in N_{0}^{T+3}
$$

is sharp therein. If we compare this sharp bound with the sharp bound for the integral of the corresponding Green's function in the continuous case of $(T+3)^{3} / 81$ in [4] then we see the bounds between the discrete and continuous cases are different. This is partly due to the differing forms of the Green's function for the discrete and continuous problems. However, it is possible to establish a connection between the two theories by forming a new bound that is common to both problems simply by choosing the larger of the two bounds. The price to pay for this unity in this situation is that one of the bounds will no longer be sharp. Thus we see a trade-off between unification and sharpness in this situation.

\section{Application of Banach's theorem}

In this section we establish sufficient conditions on the existence of a unique solution for the boundary value problem (1.1) using Banach's fixed point theorem. "The field of fixed point theory aims to establish conditions under which certain classes of problems will admit one, or more, fixed points $[21,20] "[16, \mathrm{C} 16]$. First let us recall the statement of this theorem.

Theorem 3.1 ([3]). Let $(X, d)$ be a complete metric space and $T: X \rightarrow X$ be a contraction mapping, that is, there is an $\alpha, 0 \leq \alpha<1$, such that

$$
d(T x, T y) \leq \alpha d(x, y)
$$

for all $x, y$ in $X$. Then $T$ has a unique fixed point $z$ in $X$, that is, $T z=z$. 
Every solution of the boundary value problem (1.1) can be treated as a $(T+4)$-tuple real vector. Denote the set $X=\mathbb{R}^{T+4}$ and consider the following metrics defined on $X$ :

$$
\begin{gathered}
d(u, v)=\max _{t \in \mathbb{N}_{0}^{T+3}}|u(t)-v(t)|, \\
\delta(u, v)=\left(\sum_{t=0}^{T+3}|u(t)-v(t)|^{p}\right)^{\frac{1}{p}}, \quad p>1,
\end{gathered}
$$

for all $u, v \in X$. The pair $(X, d)$ forms a complete metric space, and the pair $(X, \delta)$ also forms a complete metric space. Define the operator $T: X \rightarrow X$ by

$$
(T u)(t)=\sum_{s=2}^{T+2} G(t, s) f(s, u(s)), \quad t \in \mathbb{N}_{0}^{T+3} .
$$

Note that $u$ is a solution of the boundary value problem (1.1) if and only if $u$ is a fixed point of $T$. We apply Theorem 3.1 to show that $T$ has a unique fixed point in $X$ with the ideas manifested in the following two new theorems.

Theorem 3.2. Let $f \in C\left[\mathbb{N}_{0}^{T+3} \times \mathbb{R}, \mathbb{R}\right]$, let $f(t, 0) \neq 0$ for all $t \in \mathbb{N}_{0}^{T+3}$, let $(T+2)(T+3) \neq$ $k \eta(\eta-1)$ and let $\Gamma$ be defined in (2.14). If $f$ satisfies a Lipschitz condition with respect to the second variable on $\mathbb{N}_{0}^{T+3} \times \mathbb{R}$ with Lipschitz constant $K$, that is, there is a nonnegative constant $K$, such that

$$
|f(t, x)-f(t, y)| \leq K|x-y|, \quad \text { for all } \quad t \in \mathbb{N}_{0}^{T+3} \quad \text { and all } \quad x, y \in \mathbb{R}
$$

and

$$
K \Gamma<1,
$$

then the boundary value problem (1.1) has a unique nontrivial solution.

Proof. For $u, v \in X$ and $t \in \mathbb{N}_{0}^{T+3}$, consider

$$
\begin{aligned}
|(T u)(t)-(T v)(t)| & =\left|\sum_{s=2}^{T+2} G(t, s) f(s, u(s))-\sum_{s=2}^{T+2} G(t, s) f(s, v(s))\right| \\
& \leq \sum_{s=2}^{T+2}|G(t, s)||f(s, u(s))-f(s, v(s))| \\
& \leq K \sum_{s=2}^{T+2}|G(t, s)||u(s)-v(s)| \\
& \leq K d(u, v) \sum_{s=2}^{T+2}|G(t, s)| \\
& \leq K \Gamma d(u, v),
\end{aligned}
$$

implying that

$$
d(T u, T v) \leq \alpha d(u, v)
$$


where $\alpha=K \Gamma<1$. Thus, $T$ is a contraction mapping on $X$. Hence, by Theorem 3.1, our $T$ has a unique fixed point in $X$. This is equivalent to the boundary value problem (1.1) admitting a unique nontrivial solution. The proof is complete.

The following result sharpens the inequality (3.1) in Theorem 3.2 through the strategic use of a different metric.

Theorem 3.3. Let the conditions of Theorem 3.2 hold, with the assumption (3.1) removed. If there are constants $p>1$ and $q>1$ such that $1 / p+1 / q=1$ and

$$
K\left(\sum_{t=0}^{T+3}\left(\sum_{s=2}^{T+2}|G(t, s)|^{q}\right)^{\frac{p}{q}}\right)^{\frac{1}{p}}<1
$$

then the boundary value problem (1.1) has a unique nontrivial solution.

Proof. We apply Theorem 3.1 to show that $T$ has a unique fixed point in $X$ where $X$ is defined in the proof of Theorem 3.2 but is now coupled with the metric

$$
\delta(u, v):=\left(\sum_{t=0}^{T+3}|u(t)-v(t)|^{p}\right)^{\frac{1}{p}} .
$$

Consider

$$
\begin{aligned}
|(T u)(t)-(T v)(t)| & =\left|\sum_{s=2}^{T+2} G(t, s) f(s, u(s))-\sum_{s=2}^{T+2} G(t, s) f(s, v(s))\right| \\
& \leq \sum_{s=2}^{T+2}|G(t, s)||f(s, u(s))-f(s, v(s))| \\
& \leq K \sum_{s=2}^{T+2}|G(t, s)||u(s)-v(s)| .
\end{aligned}
$$

By Holder's inequality, we have

$$
\sum_{s=2}^{T+2}|G(t, s)||u(s)-v(s)| \leq\left(\sum_{s=2}^{T+2}|u(s)-v(s)|^{p}\right)^{\frac{1}{p}}\left(\sum_{s=2}^{T+2}|G(t, s)|^{q}\right)^{\frac{1}{q}} .
$$

Thus,

$$
\begin{aligned}
|(T u)(t)-(T v)(t)| & \leq K\left(\sum_{s=2}^{T+2}|u(s)-v(s)|^{p}\right)^{\frac{1}{p}}\left(\sum_{s=2}^{T+2}|G(t, s)|^{q}\right)^{\frac{1}{q}} \\
& \leq K\left(\sum_{s=2}^{T+2}|G(t, s)|^{q}\right)^{\frac{1}{q}} \delta(u, v)
\end{aligned}
$$

and so, we have

$$
\left(\sum_{t=0}^{T+3}|(T u)(t)-(T v)(t)|^{p}\right)^{\frac{1}{p}} \leq K\left(\sum_{t=0}^{T+3}\left(\sum_{s=2}^{T+2}|G(t, s)|^{q}\right)^{\frac{p}{q}}\right)^{\frac{1}{p}} \delta(u, v),
$$


implying that

$$
\delta(T u, T v) \leq \gamma \delta(u, v)
$$

where

$$
\gamma=K\left(\sum_{t=0}^{T+3}\left(\sum_{s=2}^{T+2}|G(t, s)|^{q}\right)^{\frac{p}{q}}\right)^{\frac{1}{p}}<1 .
$$

Thus, the conditions of Theorem 3.1 hold. Hence, by Theorem 3.1, our $T$ has a unique fixed point in $X$. This is equivalent to the boundary value problem (1.1) furnishing a unique nontrivial solution. The proof is complete.

For the choices $p=q=2$, Theorem 3.3 takes the following form:

Theorem 3.4. Let the conditions of Theorem 3.2 hold, with the assumption (3.1) removed. If

$$
K\left(\sum_{t=0}^{T+3}\left(\sum_{s=2}^{T+2}|G(t, s)|^{2}\right)\right)^{\frac{1}{2}}<1
$$

then the boundary value problem (1.1) has a unique nontrivial solution.

\section{Discussion of examples}

Let us discuss two examples to illustrate the nature of our new theorems and the relationship between them.

Example 4.1. Consider the following discrete boundary value problem

$$
\left\{\begin{array}{l}
\left(\Delta^{3} u\right)(t-2)+\frac{1}{150} \cos (u(t))=0, \quad t \in \mathbb{N}_{2}^{11}, \\
u(0)=(\Delta u)(0)=0, \quad u(12)=u(6)
\end{array}\right.
$$

We claim that this problem admits a unique solution.

Proof. Observe that (4.1) is a special case of (1.1) with $T=9, k=1, \eta=6$ and $f(t, u)=f(u)=$ $(\cos (u)) / 150$.

We show that the conditions of Theorem 3.2 are satisfied.

Since $T$ is a multiple of 3 we have $n=3$ and so $\Lambda=35$. Furthermore, appropriate calculations reveal $\Gamma \approx 146.294<147$.

Our $f$ satisfies a Lipschitz condition due to the property that its derivative with respect to $u$ is uniformly bounded by $1 / 150$ and we may choose this bound to be the Lipschitz constant, that is, on $\mathbb{R}$ we have

$$
|\partial f / \partial u|=|-\sin (u)| / 150 \leq 1 / 150=K .
$$


Finally, we see that $K \Gamma<147 / 150<1$. Thus, all of the conditions of Theorem 3.2 hold and we conclude that the discrete boundary value problem (4.1) admits a unique solution.

Let us now discuss an example that illustrates Theorem 3.3 and its distinction from Theorem 3.2.

Example 4.2. Consider the following discrete boundary value problem

$$
\left\{\begin{array}{l}
\left(\Delta^{3} u\right)(t-2)+\frac{1}{54} \tan ^{-1}(u(t))+t^{2}+1=0, \quad t \in \mathbb{N}_{2}^{11}, \\
u(0)=(\Delta u)(0)=0, \quad u(12)=u(6) .
\end{array}\right.
$$

We claim that this problem admits a unique solution.

Proof. Observe that (4.2) is a special case of (1.1) with $T=9, k=1, \eta=6$ and $f(t, u)=$ $\left(\tan ^{-1}(u)\right) / 54+t^{2}+1$.

We show that the conditions of Theorem 3.3 are satisfied with $p=2=q$, that is, Theorem 3.4 will hold.

Appropriate calculations using Maple reveal

$$
\left(\sum_{t=0}^{T+3}\left(\sum_{s=2}^{T+2}|G(t, s)|^{2}\right)\right)^{\frac{1}{2}} \approx 52.3839<53 .
$$

Our $f$ satisfies a Lipschitz condition due to the property that its derivative with respect to $u$ is uniformly bounded by $1 / 54$ and we may choose this bound to be the Lipschitz constant, that is, on $\mathbb{R}$ we have

$$
|\partial f / \partial u|=\left|1 /\left(54\left(u^{2}+1\right)\right)\right| \leq 1 / 54=K .
$$

Finally, we see that (3.6) holds since

$$
K\left(\sum_{t=0}^{T+3}\left(\sum_{s=2}^{T+2}|G(t, s)|^{2}\right)\right)^{\frac{1}{2}}<53 / 54<1 .
$$

Thus, all of the conditions of Theorem 3.3 hold with $p=2=q$ (that is, Theorem 3.4 holds) and we conclude that the discrete boundary value problem (4.2) admits a unique solution.

Remark 4.3. We note that Theorem 3.2 cannot be directly applied to the boundary value problem (4.2) in Example 4.2. The reason is because the condition $K \Gamma<1$ is not satisfied in this situation. Thus, we observe that Theorem 3.4 is more general than Theorem 3.2.

\section{Concluding remarks and further work}

This paper deepened our understanding of the existence and uniqueness of solutions to discrete boundary value problems. We showed that a larger class of problems admitted a unique solution 
and achieved this by drawing on fixed-point theory and the use of new bounds. Our results add to the recent literature on discrete boundary value problems and difference equations $[17,18]$ and move us closer to a more complete understanding of the underlying theory and application.

Although our bound on the summation of $H(t, s)$ herein is sharp, the corresponding bound involving $G(t, s)$ remains rough and a natural question for further work is: can this bound be sharpened? One of the main limitations with many fixed point theorems is the very nature of their assumptions. Because sufficient conditions are involved, it may be the case that the conditions of these theorems do not hold, yet the problem under consideration does actually admit a unique solution (or solutions). Thus it is important to also look beyond these types of sufficient assumptions and the development of new methods and altenative perspectives in mathematics are needed [21, 22] to advance the associated existence and uniqueness theory. 


\section{References}

[1] R. P. Agarwal, Difference equations and inequalities. Theory, methods, and applications, Second edition, Monographs and Textbooks in Pure and Applied Mathematics, vol. 228. New York: Marcel Dekker, 2000.

[2] R. P. Agarwal and J. Henderson, "Positive solutions and nonlinear eigenvalue problems for third-order difference equations", Comput. Math. Appl., vol. 36, nos. 10-12, pp. 347-355, 1998.

[3] R. P. Agarwal, M. Meehan and D. O'Regan, Fixed point theory and applications, Cambridge Tracts in Mathematics, vol. 141, Cambridge: Cambridge University Press, 2001.

[4] S. S. Almuthaybiri and C. C. Tisdell, "Sharper existence and uniqueness results for solutions to third-order boundary value problems", Math. Model. Anal., vol. 25, no. 3, pp. 409-420, 2020 .

[5] D. R. Anderson, "Discrete third-order three-point right-focal boundary value problems", Comput. Math. Appl., vol. 45, nos. 6-9, pp. 861-871, 2003.

[6] D. R. Anderson and R. I. Avery, "Multiple positive solutions to a third-order discrete focal boundary value problem", Comput. Math. Appl., vol. 42, nos. 3-5, pp. 333-340, 2001.

[7] D. R. Anderson and C. C. Tisdell, "Discrete approaches to continuous boundary value problems: existence and convergence of solutions", Abstr. Appl. Anal., vol. 2016, Article ID 3910972, 6 pages, 2016.

[8] M. Bohner, A. Peterson, Dynamic equations on time scales. An introduction with applications, Boston: Birkhäuser Boston-Springer, 2001.

[9] S. Elaydi, An introduction to difference equations, Third edition, Undergraduate Texts in Mathematics, New York: Springer, 2005.

[10] C. Goodrich and A. C. Peterson, Discrete fractional calculus, Cham: Springer, 2015.

[11] J. Ji and B. Yang, "Positive solutions of discrete third-order three-point right focal boundary value problems", J. Difference Equ. Appl., vol. 15, no. 2, pp. 185-195, 2009.

[12] J. Ji and B. Yang, "Computing the positive solutions of the discrete third-order three-point right focal boundary-value problems", Int. J. Comput. Math., vol. 91, no. 5, pp. 996-1004, 2014.

[13] I. Y. Karaca, "Discrete third-order three-point boundary value problem", J. Comput. Appl. Math., vol. 205, no. 1, pp. 458-468, 2007. 
[14] W. G. Kelley and A. C. Peterson, Difference equations. An introduction with applications, Second edition, San Diego-CA: Harcourt/Academic Press, 2001.

[15] S. Smirnov, "Green's function and existence of a unique solution for a third-order three-point boundary value problem", Math. Model. Anal., vol. 24, no. 2, pp. 171-178, 2019.

[16] C. P. Stinson, S. S. Almuthaybiri and C. C. Tisdell, "A note regarding extensions of fixed point theorems involving two metrics via an analysis of iterated functions", ANZIAM J. (EMAC 2019), vol. 61 (2019), pp. C15-C30, 2020.

[17] C. C. Tisdell, "On first-order discrete boundary value problems", J. Difference Equ. Appl., vol. 12, no. 12, pp. 1213-1223, 2006.

[18] C. C. Tisdell, "A note on improved contraction methods for discrete boundary value problems", J. Difference Equ. Appl., vol. 18, no. 10, pp. 1173-1777, 2012.

[19] C. C. Tisdell, "Rethinking pedagogy for second-order differential equations: a simplified approach to understanding well-posed problems", Internat. J. Math. Ed. Sci. Tech., vol. 48, no. 5, pp. 794-801, 2017.

[20] C. C. Tisdell, "Improved pedagogy for linear differential equations by reconsidering how we measure the size of solutions", Internat. J. Math. Ed. Sci. Tech., vol. 48, no. 7, pp. 1087-1095, 2017.

[21] C. C. Tisdell, "Critical perspectives of pedagogical approaches to reversing the order of integration in double integrals", Internat. J. Math. Ed. Sci. Tech., vol. 48, no. 8, pp. 1285-1292, 2017.

[22] C. C. Tisdell, "On Picard's iteration method to solve differential equations and a pedagogical space for otherness", Internat. J. Math. Ed. Sci. Tech., vol. 50, no. 5, pp. 788-799, 2019.

[23] J. Wang and Ch. Gao, "Positive solutions of discrete third-order boundary value problems with sign-changing Green's function", Adv. Difference Equ., vol. 2015, 10 pages, 2015.

[24] Y. Xu, W. Tian and Ch. Gao, "Existence of positive solutions of discrete third-order threepoint BVP with sign-changing Green's function", Adv. Difference Equ., vol. 2019, no. 206, 19 pages, 2019.

[25] Ch. Yang and P. Weng, "Green functions and positive solutions for boundary value problems of third-order difference equations", Comput. Math. Appl., vol. 54, no. 4, pp. 567-578, 2007. 